\title{
TEACHER EDUCATION: A STANDARDS-BASED ANALYSIS
}

\author{
Mahrukh Shakir ${ }^{1}$, Syed Zuhaib Aziz $^{2}$, Aman Ullah ${ }^{3 *}$, Muhammad Jawad ${ }^{4}$
}

${ }^{1}$ University of Southampton, United Kingdom; ${ }^{2}$ Department of Sociology, University of Peshawar, Pakistan; ${ }^{3 *}$ Head of Department/Lecturer, Department of Sociology, University of Swabi, Swabi, Khyber-Pakhtunkhwa, Pakistan; ${ }^{4}$ Lecturer, Department of Rural Sociology, The University of Agriculture, Peshawar, Pakistan.

Email: ${ }^{1}$ ms12g12@ southamptonalumni.ac.uk, ${ }^{2}$ zuhaib979@gmail.com, ${ }^{3 *}$ aman@uoswabi.edu.pk,

${ }^{4}$ mjawadukhel@ aup.edu.pk

Article History: Received on $21^{\text {st }}$ April 2021, Revised on $9^{\text {th }}$ May 2021, Published on $19^{\text {th }}$ May 2021

\begin{abstract}
Purpose of the study: This article aims to critically discuss the standards-based approach to language teacher education which was very popular initially in Britain and later in other parts of the world where it started to be followed as an ideal model for language teacher education. Given its popularity, a need was identified to have a critical appraisal of this popular approach for the benefit of the relevant stakeholders.

Methodology: For this purpose, this analytical article throws light on both the strengths and weaknesses of this approach through a review of other relevant empirical studies set in different educational contexts as stored in different databases and draws from a range of studies to evaluate and summarise the issues about the standards-based approach to language teacher education.
\end{abstract}

Main Findings: In light of the review findings, it is found and suggested that the standards-based approach should not have completely relied upon teacher education programs. Teacher education must be conceived of not as the experience and interpretation of a pre-determined prescribed pedagogic practice. However, if adopted in any way, the weaknesses of the standards-based approach can be mitigated if used in combination with other approaches.

Applications of this study: This study will provide stakeholders with a better understanding of the purpose and benefits of the implementation of a standards-based approach in a student-centred classroom.

The originality of this study: This study seems valuable as there exist quite limited studies, especially the recent ones, on the said topic, therefore, this study might seem to be beneficial for the relevant stakeholders who are interested in the implementation of a standards-based approach in a student-centred classroom.

Keywords: Standards-based Approach, Competency-based Approach, Language Teacher Education, Critical, Analysis.

\section{INTRODUCTION}

Although the standards-based approach may be considered appropriate in teacher education, it cannot be claimed as the bestselected model for English language teachers (Melton, 1994). The reason being, like anything else, this approach does not only possess strengths but also contains some weaknesses, both of which are the focus of the present paper. However, it is to be noted that the list of weaknesses and strengths mentioned below is not exhaustive given the scope of this paper and the available space.

Standards-based instruction and the high-stakes testing which drives it may usually be felt like a locomotive rolling over everything coming in its way, including individualised learning (Tomlison, 2000). This study seems valuable as there exist quite limited studies, especially the recent ones, on the said topic. Therefore, this study might seem to be beneficial for the relevant stakeholders who are interested in the implementation of a standards-based approach in a student-centred classroom

The paper begins with a background and behaviourist origins of standards/competence-based approach. It, then, sheds some light on the meaning and some of the main features of competency. The subsequent part presents the conceptualised meaning of the competence-based approach (henceforth CBA) in teacher education. The following last part evaluates the CBA in the light of the relevant literature and my own beliefs regarding this approach which has evolved from my learning and teaching experiences.

\section{Aims of the study}

Given the popularity of the standards-based approach, this article aims to critically discuss the standards-based approach to language teacher education. Thus, the following research questions:

1. What are the strengths of a standards-based approach?

2. What are the limitations of a standards-based approach? 


\section{METHODOLOGY}

To achieve the above-mentioned aims, a thorough review of different relevant empirical studies set in different educational contexts as stored in different databases, such as Taylor and Francis, Jstor, Eric, Sage, and Elsevier journals have been utilised. The evaluation was made, and conclusions were drawn, based on a range of studies, thereby proposing some useful suggestions.

\section{Selected Literature}

The standards/competency-based approach (henceforth CBA), identified for a large portion of the workforce, had been adopted in the 1980s, in the form of NVQs (National Vocational Qualifications), by 1992 standards of competence (Melton, 1994). Having its origin in the behaviourist movement of the 1960s and sharing most of its characteristics with the behavioural objectives, this approach was very popular in Britain during the 1980s (Melton, 1994). Thus, CBA was largely influenced by behaviourism (Hodge, 2007). It was stated that the success of a learning program needs to be assessed in terms of observable changes in the learners' behaviour (Hodge, 2007).

\section{Meaning of standards/competency}

Standards of performance are associated with the setting, specification, and realisation of the standards of performance (Melton, 1994). Standards of performance were defined in Britain under the heading of "elements and units of competence" (Melton, 1994). There exists a great deal of confusion as to what a 'competence' is (Ashworth \& Saxton, 1990) that has given rise to "a plethora of opinions about competence and its definition" (Hyland, 1991). Whether competence is about "what people can do rather than what they know (UDACE, 1989) or whether it requires to be added by the "knowledge and understanding" (Hyland, 1991).

\section{Main features of competency}

According to Martinet, the main features of the 'competency' concept are: competency occurs in a real-life setting with a set of real action variables and are exercised in a professional situation; competency is based on a set of personal resources such as skills, knowledge, and attitudes and also on the ability to mobilise those resources. In other words, knowledge of the use of these resources following their needs to take any action; the competency is a recurrent performance which implies that the efficiency and effectiveness of a competent person are not timely and temporary but stable. A competent person is always competent, capable of showing efficiency in the given situation and adapting his methods of dealing with it accordingly. Competency is more of an ongoing pursuit than an achievable target or project. Even at the highest level of competency, there is no definitive end to the prescribed goals (Gauthier, Raymond, \& Martinet, 2001).

\section{Standards/CBA in teacher education}

The idea for CBA in teacher education is that we decide certain competencies the teachers need to have and then we look for evidence of these competencies. This implies skill(s) possessed by an individual to cope effectively with job demands in a workplace (Bellis, 1997). In my understanding, 'competencies' in teacher education and so in this article, are conceptualised as a set of criteria to ensure whether certain standards are met or not. These standards are looked for/observed based on performance which does/ do not provide evidence for the presence or absence of these standards. It is a kind of normative in a sense that such and such standards are needed for teachers and that this is the correct way of teaching. Competencies or standards can be universal, or culture-based and therefore may vary with the context, place, and culture. Usually, CBA goes together with any kind of accreditation, so, for example, if someone is doing CELTA, in this course, a person i.e., the teacher educator sits and observes the trainee. The educator expects the trainee to perform certain acts, which he/she is supposed to do in the class as a teacher. The educator checks these acts, namely, the competencies e.g., in a yes/no manner or on a scale from 1 to 5. Competencies relate particularly to the desired performance in a workplace (Melton, 1994). Competency-based training is described by (Richards \& Rodgers, 2014)) as "an educational movement that advocates defining educational goals in terms of precise, measurable descriptions of the knowledge, skills, and behaviours students should possess by the end of a course of study". Course contents are stated in terms of discrete units of competency, for example, see the contents of the training courses, such as, (MACTEQ, 1994), ATESOL (2003), and CELTA. Performance indicators for the competencies most commonly used verbs, such as exhibit, design, demonstrate, work, apply, and use (Murray, 2009). In short, the main points regarding standards/competency-based approach are the following:

- Its main objective is to assess;

- There are guiding questions to look at a particular area;

- The presumption is that these are the goals, and student-teacher should work towards it and the educator judges whether a teacher is doing those things;

- The standards are set to see evidence of their performance in a student-teacher; 
- The theory is just presented to student-teachers beforehand and they get points for whether they pass or do not pass the standards.

\section{DISCUSSION}

After conducting a thorough review of the given available literature, the following strengths and limitations of the standardsbased approach have been observed. These are summarised as follows.

\section{Weaknesses of the approach}

A significant flaw of the CBA is its scope. It is restricted artificially by the imposed necessity to express competence in measurable ways (Murray, 2009). According to (C Chappell, Gonczi, \& Hager, 1995), "though it is reasonably easy to produce these sorts of descriptions when the focus is limited to relatively straightforward tasks and skills needed in the workplace, attempts to produce descriptions of more complex work practices, commonly involving complicated interactions of various sets of knowledge, skills, and abilities, generally, fail". Discussing the narrow conceptions of CBA, Toohey, Ryan, McLean, and Hughes (1995) criticised the CBA for its tendency of assessing only those areas of performance that are of a routine and are easy to measure and emphasised the need of going beyond the mere technical skills.

CBA is excessively reductionist, rigid, and narrow (Clive Chappell, 1996; Hyland, 1994). Competence is defined in terms of "the performance of discrete tasks, identified by functional analysis of work roles [which] is the basis for [standards or] competency statements upon which competence is assessed (Kerka, 1998). Blunden (1996) argues that operationalization of abstract or/and complex tasks into measurable discrete units may trivialize the craft inherent in many tasks. CBA emphasizes knowing about teaching instead of doing teaching (NRGTSP, 2003). Standard statements cannot capture the complexity of practice (NRGTSP, 2003).

It is also argued that reductionist competencies and behaviourist assessment encourage trainees to simply reproduce learned knowledge, which thus leads to a compromise of depth and complexity. These standards can exert rather rigid control over student teachers to make sure employer's wishes are met (Lucas, 2004). Thus, there is a possibility of trainees being aware of the competencies required of them and so they may fake or articulate them in front of the teacher educators.

Cultural issues are also not addressed in this approach. According to Mulcahy and James (2000), there may occur different and probably contradictory cultures and concepts of competence. Owing to the varying number of cultures and contexts where the trainee needs to demonstrate performance, it is usually not practically possible to measure the trainees' performance within all the possible contexts and to ensure whether the trainee may be able to transfer to the other occasions the skills or competencies demonstrated on one occasion (though may be possible for simple closely related situations (Evans, 1995). Disagreements exist about the existence of such context-free attributes, the transferability of these attributes, and the attempt to describe understanding, knowledge, attitudes, and cognition as behavioral objectives when they are not behaviors (Gonczi, 1997).

The competencies fragment, making technical, and de-contextualise work of the teachers (Louden \& Wallace, 1993). This de-contextualization of competencies stands in contrast to the actual real context which includes all the variables of the profession concerned, and there exist real-world constraints which come into play; a fact ignored in teacher education (Gauthier et al., 2001). For a person to be qualified as competent, in addition to containing skills, knowledge, and attitudes from his pool of resources, competency also needs an additional element, that is, a context, which implies given space and time, and not only controlled situations (Gauthier et al., 2001). The condition of context suggests that a competent person must have the potential of; recognizing the constraints and demands of the situation, identifying the available resources, and taking action by bringing into play those resources in such a way as to be effective and relevant to the given situation (Gonczi, 1997). This ability to act promptly requires quick judgment and presence of mind. Competency, therefore, does not lie in the ability to apply but to construct, read a situation, give it a meaning and deal with it (Gauthier et al., 2001).

In real classrooms, the roles played by teachers are mostly unpredictable, and the circumstances which trigger them is not possible to be reproduced by a competency-based trainer equipped with a pre-determined, de-contextualised checklist (Murray, 2009).

CBA, having its main focus on technical competence, neglects other social, emotional, and intellectual skills. This fact has been acknowledged by the NCVER (2001) as, CBA "is not as well suited to the development of conceptual and experiential knowledge" though "it seems effective for routine problem-solving skills, making it well suited for technical skills acquisition" Biggs (1994) also advocates the process over the products of learning and identified risk of ignoring psychological issues in the quantitative approach followed in CBA.

The standards-based approach entails technicality, that is, actions and ideas are locked in excessive precision. There seems 
no logic in substituting lists of skills and knowledge by a competency framework if this framework is just another way to describe the same (Gauthier et al., 2001).

Another problem with such an approach is that standards (or any other prescriptive code) can be subjected to interpretation in many ways, and thus likely not leading to a commonality of practice (Lucas, 2004). Human judgments are subjective and the identified competencies reflect the judgment of the educators involved in the process. The judges working independently is very likely to arrive at different conclusions (Melton, 1994). CBA entails the difficulty and risk of reaching an agreement on standards (Murray, 2009). Thus, the effective exercise of discretion requires greater expertise and professionalism on the part of educators. Also, using standards for assessing trainees may lead to different interpretations of the standards even by the trainees according to their experience of previous work and of teaching in subject traditions (Lucas, 2004).

The "checklist" approach, in which an individual can/cannot perform a specific task, or competency is achieved/not achieved is viewed as de-motivating, and simplistic, which suggests a "minimum" level of acceptable performance instead of a standard of excellence (Blunden, 1996).

Defining the work of teachers in terms of competencies deskills teachers and, instead of their practices being transformative, reinforces them as reproductive of schooling (Porter, Rizvi, Knight, \& Lingard, 1992). Thus, the CBA contains the tendency to limit the professional growth of teachers, instead of extending and transforming it (Whitty, 1994). Identifying standards and measuring the related performance may not necessarily lead to the realisation of set standards without motivating trainees to achieve the prescribed standards by making them realise their importance and relevance and that they are achievable (Evans, 1995).

There are no standards for measuring standards, and to escape this complexity, the educators may resort to the comfort zone of competency statements and ticking boxes (Lucas, 2004).

By breaking down the objectives and qualifying the conditions of performance, the list gets impossibly long. Where competencies are taken as the sole requirement to be met, the units of competence - fragmented into very precise detail of what trainees are expected to achieve - seem to leave very little room for the trainees to determine their goals and how to achieve them (Melton, 1994).

The student teachers are very likely to feel quite constrained by the standards which are specifications and more of a checklist, which determine everything they do rather than to ensure that they cover and include what is required. The setting of standards may create considerable stress if the trainees feel threatened by the imposition of such standards especially if they feel that they may suffer in some form if they are unable to achieve the set standards (Evans, 1995).

The behavioral nature of CBA in teacher education is negatively viewed for its failure to foster the development of broader skills required for the profession concerned. In this approach, "the issues may at times be fudged by the need to compromise and findings may be reduced to the common denominator underlying a variety of different perceptions" (Evans, 1995). The standards are set in response to perceived needs at a specific point in time and reflect the perceptions of a specific person or group and so are opened to change with time with the change of perceptions and needs (Evans, 1995).

Measurement of standards or analysis of performance tends to be more focused on isolated skills. Mastery of the different elements of competence may not necessarily automatically lead to the acquisition of more complex skills which could be based upon factors other than the ones identified ((Evans, 1995): 289).

Formulation of competencies implies more of adopting a high level of abstraction than simply listing the skills and performances to be mastered (Gauthier et al., 2001). Competencies can be formulated so generally as to leave the competency statements no more than simply a series of empty formulas, which may not guide thinking and offer no direction whatsoever for the preparation of training programs and also no support for training providers. So formulation of competency statements requires care in terms of defining the kind of professional to be trained (Gauthier et al., 2001).

"Teaching by objectives, which reduced learning to the realisation of a set of behavioural objectives, led to such a level of fragmentation that the students were no longer able to understand the meaning of what they were being asked to do, and it was by no means certain that the set of behaviours learned coincided with the objectives they were supposed to constitute" (Rey 1998 as mentioned in Gauthier et al. (2001).

In addition to the above-mentioned weaknesses, other flaws in the standards-based approach, according to my opinion, are;

- It fails to account for the variation in the teaching styles of individual teachers. CBA constraints individualisation restricts creativity and proposes a "one size fits all" ideal.

- Beginners and mid-career teachers bring their talents to the programme and these talents if do not fall in the scope of the standards, then instead of help, these standards harm. 
- Standards are normally generalized statements; their specification imposes a limitation on the achievement of standards.

- There is less room for student-teachers perspectives.

- Feedback can be confrontational as teacher educators just look at outcomes in terms of seeing/not seeing them. Trainees may feel offensive about it.

- Good behaviours are usually taken for granted by educators.

- Usually, people feel more pressure in being assessed as compared to just being observed.

- CBA is not developmentally supportive. For instance, student-teachers have done this, either tick or cross; their problems are theirs, it does not teach e.g., how to teach a grammar point.

\section{The Strengths are as follows}

Given the work that teachers perform, they can be described as professionals. This implies the organisation of the skills, attitudes, and knowledge, specific to the profession concerned, and the consolidation and development of the competencies required for practising a profession (Martinet et al., 2001: 17). The professionalisation of an occupation implies ascertaining that any practitioner reaches a specified level of competence and should be aware of acting correctly. At an individual level, professionalisation means to be able to combine techniques, knowledge, strategies, and attitudes to perform particular tasks (Martinet et al., 2001). Professionalisation depends on transmissible knowledge or codified practical knowledge. This codification helps in acquiring the same skills and knowledge, shared by the practitioners, which can be transmitted by training (Martinet et al., 2001: 18).

CBA may enable trainees to better understand the nature of their work provided in the form of descriptors and to articulate the requirements of it (Bowden \& Masters, 1993). It has a high validity by having a direct bearing on significant aspects of the job (Van der Merwe \& Potgieter, 2002) and provides opportunities to individuals to "achieve qualifications that relate to the required performance in the workplace" (Erridge \& Perry, 1994).

CBA may provide a framework in which administrators and student-teachers become accountable for quality (Jackson, 1993). CBA may be perceived as having inherent fairness since it focuses on behaviour, rather than culture or personality factors, and fairness is linked with value judgements, operationalised in actions or decisions which relies upon assessment scores (Van der Merwe \& Potgieter, 2002).

CBA facilitates and promotes effective information sharing related to professional teaching practice and it establishes and provides the agreed foundational elements and dimensions of effective teaching (NFST, 2003). Standards prescribed for teaching capture key features of teachers' work and describe the skills and knowledge for effective teaching. Standards make explicit a good teaching practice and provide consistency in respect of what constitutes quality teaching and, through the development of standards, facilitate the knowledge and skills construction and understandings for effective teaching (NRGTSP, 2003).

The set standards provide a common framework to exchange dialogue between teacher educators and student-teachers and also provide guidance and support for teachers' professional development (NRGTSP, 2003). The use of standards may help in improving dialogue between teachers and educators about performance and teaching expectations (Heneman III, Milanowski, Kimball, \& Odden, 2006).

CBA provides a standardised set of activities and so is more streamlined, less time-consuming (usually not more than a few hours), and normally does not involve multiple observers (Van der Merwe \& Potgieter, 2002).

Prescribing standards for teaching helps in making teachers' capabilities and knowledge, explicit for the ones outside and within the profession, and offers means by which good teaching may be identified, celebrated, and rewarded (Van der Merwe \& Potgieter, 2002).

Setting standards and engagement with teachers may help them in planning, structuring, and facilitating their ongoing professional learning (Mayer, Mitchell, Macdonald, Land, \& Luke, 2003).

Describing standards serve as recognisable and common points of reference for professional engagement, and provides a framework to which teachers may aspire (NFST, 2003).

Standards of competency may enhance the teaching quality by laying down what reckons as competence, as a foundation for delivering and developing both pre-and in-service training and also for taking decisions concerning entry to the teaching profession and for progress in the career (Louden \& Wallace, 1993). 
Developing a set of teaching standards and scoring rubrics (that is, a competency model) reflects what teachers are expected to know and to do to provide the type of instruction required to meet the students' achievement goals. Teachers get a clear and concise understanding of the expectations from them for their instructional practice (Heneman III et al., 2006).

In a standards-based approach, the teaching standards and the explicit descriptors for teachers' performance may help the teacher educators to do a better job as evaluators and also has the potential for teachers to get useful feedback for improving their performance (Heneman III et al., 2006).

Because evaluation is more individualised, assessment can be more closely tied to what is needed and meet individual learners' weaknesses and strengths, thus determining learners' needs (Glendenning, 1983).

Other strengths, according to my opinion, could be;

- Clear objectives set to achieve in the training programme,

- Ease in management of the teacher training,

- Ease in the evaluation of the training outcomes,

- Comparability of the course contents and teacher's skill with agreed standards.

\section{CONCLUSION}

This paper argues that although the competency-based approach is relatively convenient in terms of clear descriptors, assessment, and management while carrying a certain level of objectivity, however, the basic flaws in the model, such as decontextualisation, subjective judgment, particularly the emphasis upon technical competence, and the criterion of measurability makes CBA "unsuitable as an approach to course design and evaluation in second language teacher education". (Murray, 2009: 19). Some concerns were also shown regarding the competence of the evaluator; leniency, and strictness, though some teachers may also find this approach quite stressful. The weaknesses of CBA can be mitigated if used in combination with other approaches and not to be fully relied on in teacher education programs. According to Kumaravadivelu (2001) as cited in Murray (2009);

"Teacher education must be conceived of not as the experience and interpretation of a pre-determined prescribed pedagogic practice, but rather as an ongoing dialogically constructed entity involving two or more critically reflective interlocutors"

\section{APPLICATION OF THE STUDY}

Thus, this study is an attempt to provide the relevant stakeholders with a better understanding of the purpose, benefits, and shortcomings of the implementation of a standards-based approach in a student-centred classroom so that they may take necessary actions accordingly in their relative spheres.

\section{LIMITATION AND STUDY FORWARD}

Even though a great effort has been made to include as many relevant studies as possible to reach a sound and thoroughly justified analysis of the given topic, however, due to space limitations, it might not have been possible to include all those studies. Moreover, to my knowledge, since limited work has been carried out on this topic, especially from around 2007 onwards, so most of the studies referred to in this article belongs to the time span before 2007. As empirical research on this topic seems to have been limited, particularly recently, more will be required and fruitful as the teaching and learning situation changes with time.

\section{ACKNOWLEDGEMENT}

During this study, there was no conflict of interest among co-authors. We declare that all the work presented in this work is solely our own. Further, this study is not supported by any funding agency.

\section{AUTHORS CONTRIBUTION}

The conception and design of the article were presented by Dr. Mahrukh Shakir. Critical revision of the article for important intellectual content was done by Dr. Syed Zuhaib Aziz. Mr. Aman Ullah did a lot of work in references, citations, and structure of the paper. Dr. Muhammad Jawad Critically evaluated the final draft. All authors discussed the results and contributed to the final manuscript.

\section{REFERENCES}

1. Ashworth, P. D., \& Saxton, J. (1990). On 'Competence'. Journal of Further and Higher Education, 14(2), 3-25. https://doi.org/10.1080/0309877900140201 
2. ATESOL. (2003). Draft NSW professional teaching standards working draft for consultation. Association of Teachers of English to speakers of other languages.

3. Bellis. (1997). An interpretation of competence. People Dynamics. 15(1), 32-33. People Dynamics, 15(1), 32-33.

4. Biggs, J. (1994). Learning outcomes: competence or expertise? Australian and New Zealand Journal of Vocational Education Research, 2(1), 1-18.

5. Blunden, R. (1996). The Mind Dependency of Vocational Skills. Journal of Vocational Education \& Training, 48(2), 167-188. https://doi.org/10.1080/1363682960480205

6. Bowden, J. A., \& Masters, G. N. (1993). Implications for higher education of a competency-based approach to education and training.

7. Chappell, C. (1996). Quality \& competency based education and training. Paper presented at the The Literacy Equation: Competence $=$ Capability? National Conference of the Australian Council for Adult Literacy Conference Papers (Queensland, Australia, November 7-9.

8. Chappell, C., Gonczi, A., \& Hager, P. (1995). Competency-based education. In G. Foley (Ed.), Understanding adult education and training Sydney: Allen \& Unwin.

9. Erridge, A., \& Perry, S. (1994). The Validity and Value of National Vocational Qualifications: the case of purchasing. The Vocational Aspect of Education, 46(2), 139-154. https://doi.org/10.1080/0305787940460204

10. Evans, K. (1995). Competence and Citizenship: Towards a Complementary Model for Times of Critical Social Change. British Journal of Education \& Work, 8(2), 14-27. https://doi.org/10.1080/0269000950080202

11. Gauthier, C., Raymond, D., \& Martinet, M. A. (2001). Teacher training: orientations, professional competencies (State or province government publication: English ed.). Québec (Province). Ministère de l'éducation: Ministère de l'éducation, (2001.

12. Glendenning, D. (1983). Competency-based education and lifelong learning. Canada.

13. Gonczi, A. (1997). Future Directions for Vocational Education in Australian Secondary Schools. Australian and New Zealand Journal of Vocational Education Research, 5(1), 77-108.

14. Heneman III, H. G., Milanowski, A., Kimball, S., \& Odden, A. (2006). Standards-based teacher evaluation as a foundation for knowledge and skill-based pay. Retrieved from https://doi.org/10.12698/cpre.2013.rb45

15. Hodge, S. (2007). The Origins of Competency-Based Training. Australian Journal of adult learning, 47(2), 179209.

16. Hyland, T. (1991). Professionalism, competence and teaching in post-school education. education and training, 45.

17. Hyland, T. (1994). Competence, education and NVQs: Dissenting perspectives: Cassell London.

18. Jackson, N. (1993). If competence is the answer, what is the question? Australian and New Zealand Journal of Vocational Education Research, 1(1), 46-60.

19. Kerka, S. (1998). Competency-Based Education and Training. Myths and Realities.

20. Louden, W., \& Wallace, J. (1993). Competency standards in teaching: exploring the case. Unicorn, 19(1), 45-53.

21. Lucas, N. (2004). Using work-based learning to rethink initial teacher education programmes for adult and further education teachers: Beyond a standards-based model and the importance of knowledge. The University of London.

22. MACTEQ. (1994). Desirable attributes of beginning teachers. Sydney.

23. Mayer, D., Mitchell, J., Macdonald, D., Land, R., \& Luke, A. (2003). From personal reflection to the professional community: Education Queensland Professional Standards for Teachers Evaluation of the 2002 Pilot. Brisbane, Queensland: Department of Education. Retrieved April 15, 2004.

24. Melton, R. F. (1994). Competences in perspective. Educational Research, 36(3), 285-294. https://doi.org/10.1080/0013188940360307

25. Mulcahy, D., \& James, P. (2000). Evaluating the contribution of competency-based training: an enterprise perspective. International Journal of Training and Development, 4(3), 160-175. https://doi.org/10.1111/14682419.00105

26. Murray, J. (2009). Teacher competencies in the post-method landscape: The limits of competency-based training in TESOL teacher education.

27. NCVER. (2001). Ausralian Apprenticeships. Facts, and Future Adelaide.

28. NFST. (2003). A consultative paper. Melbourne: : Melbourne: TQELT Secretariat.

29. NRGTSP. (2003). Statement from the Teaching Profession on Teacher Standards Quality and Professionalis. Canberra: Australian College of Educators.

30. Porter, P., Rizvi, F., Knight, J., \& Lingard, R. (1992). Competencies for a clever country: Building a house of cards? Unicorn, 18(3), 50-58.

31. Richards, J. C., \& Rodgers, T. S. (2014). Approaches and methods in language teaching: Cambridge university press.

32. Tomlison, C. A. (2000). Reconclable Difference? Standart based teaching and Differentitation (Vol. 58). 
33. Toohey, S., Ryan, G., McLean, J., \& Hughes, C. (1995). Assessing competency-based education and training: a literature review. Australian and New Zealand Journal of Vocational Education Research, 3(2), 86-117.

34. UDACE. (1989). Understanding competence: a development paper. Leicester Retrieved from NIACE.

35. Van der Merwe, R., \& Potgieter, T. (2002). Assessment in the workplace: a competency-based approach. $S A$ Journal of industrial psychology, 28(1), 60-66. https://doi.org/10.4102/sajip.v28i1.31

36. Whitty, G. (1994). Deprofessionalising teaching?: Recent developments in teacher education in England. 